Quark deconfinement phase transition in nuclear matter for improved quark mass densitydependent model

This content has been downloaded from IOPscience. Please scroll down to see the full text. 2012 EPL 9821001

(http://iopscience.iop.org/0295-5075/98/2/21001)

View the table of contents for this issue, or go to the journal homepage for more

Download details:

IP Address: 200.239.167.219

This content was downloaded on 10/02/2015 at 20:45

Please note that terms and conditions apply. 


\title{
Quark deconfinement phase transition in nuclear matter for improved quark mass density-dependent model
}

\author{
Chen Wu ${ }^{1(a)}$, Wei-Liang Qian ${ }^{2}$, Yu-Gang MA ${ }^{1}$, Guo-Qiang Zhang ${ }^{1}$ and Sanjeev KumaR ${ }^{1}$ \\ ${ }^{1}$ Shanghai Institute of Applied Physics, Chinese Academic of Sciences - Shanghai 201800, China \\ ${ }^{2}$ Universidade de Ouro Preto - Ouro Preto, 35400-000, Brazil
}

received 10 February 2012; accepted in final form 19 March 2012

published online 20 April 2012

PACS 11.10.Wx - Finite-temperature field theory

PACS 12.39. Ba - Bag model

PACS 25.75.Nq - Quark deconfinement, quark-gluon plasma production, and phase transitions

\begin{abstract}
The improved quark mass density-dependent (IQMDD) model, which has been successfully used to describe the properties of both infinite nuclear matter and finite nuclei, is applied to investigate the properties of quark deconfinement phase transition. By using the finite temperature quantum field theory, we calculate the finite temperature effective potential and extend the IQMDD model to finite temperature and finite nuclear matter density. The critical temperature and the critical density of nuclear matter are given and the QCD phase diagram is addressed. It is shown that this model can not only describe the saturation properties of nuclear matter, but also explain the quark deconfinement phase transition successfully.
\end{abstract}

Copyright (C) EPLA, 2012

Owing to the nonperturbative nature of quantum chromodynamics (QCD) in low-energy regions, it is very difficult to study a nuclear system by using QCD directly. Phenomenological models reflecting the characteristic of the strong interaction are widely used in the study of the properties of hadrons and nuclear matter. The quark-meson coupling (QMC) model suggested by Guichon [1] is a famous hybrid quark meson model, which can successfully describe the saturation properties of nuclear matter and many other properties of finite nuclei $[2,3]$. In this model, the nuclear system was suggested as a collection of MIT bag, vector $\omega$-meson and scalar $\sigma$-meson, and the interactions between quarks and mesons are limited within the MIT bag regions due to the fact that the quark cannot escape from the MIT bag.

Although the QMC model is successful for describing the physical properties of a nuclear system, two shortcomings arise when one uses this model to discuss some physical topics. First, the MIT bag model is a permanent quark confinement model and its boundary condition cannot be destroyed by temperature and nuclear matter density. The second difficulty arises from the MIT boundary condition. If we hope to do the nuclear many-body calculations beyond mean-field approximation (MFA) by quantum field theory, it is essential to find the free

(a) E-mail: wuchenoffd@gmail.com propagators of quark, $\sigma$-meson and $\omega$-meson, respectively. However, the constraint of the MIT bag boundary condition presents obstacles to get the corresponding propagators in free space, because the interactions between quarks and mesons are limited within the bag regions, and multireflection of quarks and mesons by the boundary must be taken into account [4].

In order to keep the quark confined property and give up the MIT boundary condition, a new quark-meson coupling model based on a quark mass density-dependent (QMDD) model was presented in our previous papers $[5,6]$. According to the QMDD model, the masses of $u, d$ and $s$ quarks (and the corresponding antiquarks) satisfy

$$
\begin{aligned}
m_{q} & =\frac{B}{3 n_{B}} \quad(q=u, d, \bar{u}, \bar{d}), \\
m_{s, \bar{s}} & =m_{s 0}+\frac{B}{3 n_{B}},
\end{aligned}
$$

where $n_{B}=\frac{1}{3}\left(n_{u}+n_{d}+n_{s}\right)$ is the baryon number density, with $n_{u}, n_{d}, n_{s}$ representing the density of the $u$-quark, $d$-quark, and $s$-quark, $m_{s 0}$ is the current mass of the strange quark, and $B$ is the vacuum energy density inside the bag. As was explained in ref. [7], the ansatz in eqs. (1) and (2) corresponds to a quark confinement hypothesis because when $V \rightarrow \infty, n_{B} \rightarrow 0$ and $m_{q} \rightarrow \infty$, it prevents the quark from going to infinity or to very large regions. 
The large volume means that the baryon density is small. This mechanism of confinement can be mimicked through the requirement that the mass of an isolated quark becomes infinitely large so that the vacuum is unable to support it [8]. This is just the physical picture given by eqs. (1) and (2). In fact, this confinement mechanism is very similar to that of the MIT bag model [9]. But the advantage of the QMDD model is that it does not need to introduce a quark confined boundary condition like that of the MIT bag model.

But the QMDD model still has two shortcomings: 1) It is still an ideal quark gas model. No interactions between quarks exist except for a confinement ansatz, eqs. (1) and (2). 2) It cannot describe the quark deconfinement phase transition and predict a correct phase diagram as that given by lattice QCD. The reason is that the temperature $T$ tends to infinity when density $n_{B} \rightarrow 0$. This result can easily be understood if we notice the basic hypothesis, eqs. (1) and (2), of the QMDD model, and the quark masses are divergent when $n_{B} \rightarrow 0$. To excite an infinite weight particle, one must prepare to pay the price for infinite energy, i.e., infinite temperature. It means that the confinement in the QMDD model is still permanent. To overcome the difficulty 2 ), we have introduced a new ansatz, i.e., that the vacuum density $B$ is a function of temperature $T[10]$ :

$$
\begin{aligned}
& B(T)=B_{0}\left[1-\left(T / T_{C}\right)^{2}\right], 0 \leqslant T \leqslant T_{C}, \\
& B(T)=0, T>T_{C} .
\end{aligned}
$$

and extended the QMDD model to a quark mass densityand temperature-dependent (QMDTD) model [10]. This QMDTD model has been employed to discuss the properties of strange quark matter [11], the dibaryon system [12] successfully.

To improve the shortcoming 1) of the QMDD model and to avoid the ad hoc ansatz of the QMDTD model, we introduced the $\omega$-meson and $\sigma$-meson in the QMDD model to mimic the repulsive and attractive interactions between quarks in refs. [5,6]. Following the treatment of Friedberg and Lee $[13,14]$, the interaction between quarks and the nonlinear $\sigma$-field forms a Friedberg-Lee bag [5]. In ref. [5], we found the wave functions of the quark ground state and the lowest one-quark excited states. By using these wave functions, we calculated many physical quantities such as the root-mean-square radius, the magnetic moment of a nucleon to compare with experiments and come to the conclusion that this IQMDD model is successful to explain the properties of a nucleon. Then under mean-field approximation, we employ the IQMDD model to investigate the physical properties of nuclear matter. Instead of the MIT bag, after introducing the nonlinear interaction of $\sigma$-mesons and $q q \sigma$ coupling, we construct a Friedberg-Lee soliton bag in a nuclear system. The quark and $\sigma$-meson coupling and the quark and $\omega$-meson coupling are introduced to mimic the attractive and the repulsive interactions between quarks in this model. It has been shown that our model can successfully describe the saturation properties, the equation of state, the compressibility and the effective nucleon mass of symmetric nuclear matter [6] and finite nuclei [15].

We hope to emphasize that there are two basic differences between the IQMDD model and the usual QMC model. First, unlike in the QMC model, we do not need a MIT bag for the nucleon in the IQMDD model. The constraint of the MIT bag boundary condition disappears in our formulas because the quark confinement mechanism has been established in eqs. (1) and (2). Second, the interaction between the quark and the scalar meson is limited in the bag regions for the QMC model, while for the IQMDD model this interaction is extended to the whole free space. So it is convenient to write down the free propagators of quarks and mesons.

In refs. $[16,17]$, we extended this model to a finite temperature and studied its soliton solution by means of the finite temperature quantum field theory. The critical temperature of quark deconfinement $T_{C}$ and the function of the temperature-dependent bag constant $B(T)$ are found as an output. But the the deconfinement properties of the IQMDD model at finite density of nuclear matter and the QCD phase diagram have not been studied yet. We would like to study these problems in this paper. We will show that we can get reasonable quark deconfinement critical temperature and critical density of nuclear matter. Moreover, the QCD phase diagram can also be given in the IQMDD model phenomenologically.

Before giving the main formulas of the IQMDD model, we should emphasize that the quark deconfinement phase transition and the QCD phase diagram have attracted a lot of interest for many years many theoretical efforts have been recently and less recently carried out, on the study of the hadronic phase structure and the deconfinement phase transition by using the Nambu-Jona-Lasinio model and some of its extensions in the framework of finite temperature field theory [18-29]. Among them, some perform a very detailed investigation of the QCD phase structure and other ones give precise numerical estimates for the deconfinement temperature and size of hadrons. These publications are very interesting and also give us lots of important information for the QCD phase structure.

The effective Lagrangian density of the IQMDD model is given by

$$
\begin{aligned}
\mathcal{L}= & \bar{\psi}\left[i \gamma^{\mu} \partial_{\mu}-m_{q}-g_{\sigma}^{q} \sigma-g_{\omega}^{q} \gamma^{\mu} \omega_{\mu}\right] \psi \\
& +\frac{1}{2} \partial_{\mu} \sigma \partial^{\mu} \sigma-U(\sigma)-\frac{1}{4} F_{\mu \nu} F^{\mu \nu}+\frac{1}{2} m_{\omega}^{2} \omega^{\mu} \omega_{\mu}
\end{aligned}
$$

where $F_{\mu \nu}=\partial_{\mu} \omega_{\nu}-\partial_{\nu} \omega_{\mu}, \psi$ represents the quark field, $m_{q}=\frac{B}{3 n_{B}}$ is the mass of $u(d)$-quark, the $\sigma$ and $\omega$ fields are not dependent on time, $g_{\sigma}^{q}$ is the coupling constant between the quark field $\psi$ and the scalar meson field $\sigma, g_{\omega}^{q}$ is the coupling constant between the quark field $\psi$ and the vector meson field $\omega_{\mu}, U(\sigma)$ is the self-interaction potential 
for the $\sigma$-field. The potential field $U(\sigma)$ is chosen as [14]

$$
\begin{gathered}
U(\sigma)=\frac{a}{2 !} \sigma^{2}+\frac{b}{3 !} \sigma^{3}+\frac{c}{4 !} \sigma^{4}+B, \\
b^{2}>3 a c .
\end{gathered}
$$

The condition (7) ensures that the absolute minimum of $U(\sigma)$ is at $\sigma=\sigma_{v} \neq 0$. The potential $U(\sigma)$ has two minima: one is the absolute minimum $\sigma_{v}$,

$$
\sigma_{v}=\frac{3|b|}{2 c}\left[1+\left[1-\frac{8 a c}{3 b^{2}}\right]^{\frac{1}{2}}\right]
$$

it corresponds to the physical vacuum, and the other is at $\sigma_{0}=0$, it represents a metastable local false vacuum. We take $U\left(\sigma_{v}\right)=0$ and the bag constant $B$ can be expressed as

$$
-B=\frac{a}{2 !} \sigma_{v}^{2}+\frac{b}{3 !} \sigma_{v}^{3}+\frac{c}{4 !} \sigma_{v}^{4}
$$

In order to study the deconfinement phase transition, we turn to extend the IQMDD model to finite temperature. The appropriate framework is the finite temperature quantum field theory. The finite temperature effective potential plays a central role within this framework. Under the mean-field approximation, the $\omega$-meson field operator can be replaced by its expectation value in symmetric nuclear matter [30],

$$
\bar{\omega}_{\mu}=\delta_{\mu 0} \bar{\omega}=\delta_{\mu 0} \frac{3 g_{\omega}^{q}}{m_{\omega}^{2}} \rho_{B} .
$$

Using the method of Dolan and Jackiw [31,32], up to one-loop approximation, the effective potential reads

$$
V\left(\sigma ; \beta ; V_{\omega}\right)=U(\sigma)+V_{B}(\sigma ; \beta)+V_{F}\left(\sigma ; \beta ; V_{\omega}\right)
$$

where

$$
V_{\omega}=g_{\omega}^{q} \bar{\omega}=3 \frac{g_{\omega}^{q 2}}{m_{\omega}^{2}} \rho_{B}
$$

is the contribution of the $\omega$-field, $\beta$ is the inverse of the temperature, $\rho_{B}$ is the density of nuclear matter and

$$
\begin{aligned}
V_{B}(\sigma ; \beta)= & \frac{1}{2 \pi^{2} \beta^{4}} \int_{0}^{\infty} \mathrm{d} x x^{2} \ln \left(1-e^{-\sqrt{\left(x^{2}+\beta^{2} m_{\sigma}^{2}\right)}}\right), \\
V_{F}\left(\sigma ; \beta ; V_{\omega}\right)= & -12 \sum_{n} \frac{1}{2 \pi^{2} \beta^{4}} \\
& \int_{0}^{\infty} \mathrm{d} x x^{2} \ln \left(1+e^{-\left(\sqrt{\left(x^{2}+\beta^{2} m_{q}^{2}\right)}-\beta V_{\omega}\right)}\right),
\end{aligned}
$$

where the minus sign of eq. (14) is the consequence of Fermi-Dirac statistics. The degenerate factor 12 comes from: 2 (particle and antiparticle), 2 (spin), 3 (color). $m_{\sigma}$ and $m_{q}$ are the effective masses of the scalar field $\sigma$ and the quark field, respectively, which can be found in refs. $[16,17]$.
We see from eqs. (11)-(14) that the scalar-like interaction $g_{\sigma}^{q} \psi^{+} \sigma \psi$ gives a contribution for effective masses of quark and $\sigma$-meson and then forms a confined soliton bag, while the vector-like interaction $g_{\omega}^{q} \psi+\gamma^{\mu} \omega_{\mu} \psi$ gives a contribution to an effective chemical potential of quarks. In fact, this finite temperature effective potential for the Friedberg-Lee (FL) model had been calculated by many other authors $[16,17]$.

In the FL bag model, the finite temperature vacuum energy density $B(T)$ is defined as

$$
B\left(\beta ; V_{\omega}\right)=V\left(\sigma_{0} ; \beta ; V_{\omega}\right)-V\left(\sigma_{v} ; \beta ; V_{\omega}\right) .
$$

It is the difference between the values in the perturbative false vacuum state and the values in the physical real vacuum state of the finite temperature effective potential. At critical density of nuclear matter and critical temperature of quark deconfinement phase transition, $B$ is equal to zero: $B\left(T, \rho_{B}\right)=0$.

Before numerical calculations, let us discuss the parameters in the IQMDD model. First, we choose $m_{\omega}=783 \mathrm{MeV}, m_{\rho}=770 \mathrm{MeV}$ and $m_{\sigma}=509 \mathrm{MeV}$ as those of ref. [6]. Fixing the nucleon mass $M_{N}=939 \mathrm{MeV}$, we get $B=174 \mathrm{MeV} \mathrm{fm}^{-3}$. Obviously, the behaviors at the saturation point must be explained for a successful model. It reveals that nuclear matter saturates at a density $\rho_{0}=0.15 \mathrm{fm}^{-3}$ with a binding energy per particle $E / A=-15 \mathrm{MeV}$ at zero temperature, and the compression constant is about $K\left(\rho_{0}\right)=210 \mathrm{MeV}$. Therefore, we fixed $g_{\omega}^{q}=2.44, g_{\sigma}^{q}=4.67, b=-1460 \mathrm{MeV}$ to explain the above data [6].

Now we turn to investigate the effective potential $V(\sigma$; $\left.\beta ; V_{\omega}\right)$. The effective potential at finite temperature and finite nuclear matter density can be obtained by numerical calculations using the set of eqs. (11)-(14). Then it is convenient to investigate the temperature and the nuclear matter density dependence of the bag constant. The bag constant in the IQMDD model is defined as eqs. (15). The upper panel of fig. 1 shows the temperature-dependent behavior of the bag constant at various nuclear matter densities. One can clearly see that the bag constant decreases continuously with increasing temperature at fixed nuclear matter density. At critical temperature $T_{C}$, the bag constant equals zero and the deconfinement phase transition begins to occur. The lower panel of fig. 1 shows the nuclear matter density dependence of the bag constant at different temperatures. Similarly as in the upper panel of fig. 1, one can see that the deconfinement phase transition begins to take place at a critical density for a given value of temperature.

The critical behavior of nuclear matter in the $T-\rho_{B}$ phase diagram calculated using the IQMDD model is described in fig. 2. The critical line corresponds to the phase transition between confined and deconfined phases. In this nuclear model, our calculation shows that the critical density of the nuclear matter for quark deconfinement is about ten times the saturation density of the nuclear matter. The phase diagram shown in fig. 2 , based on the 

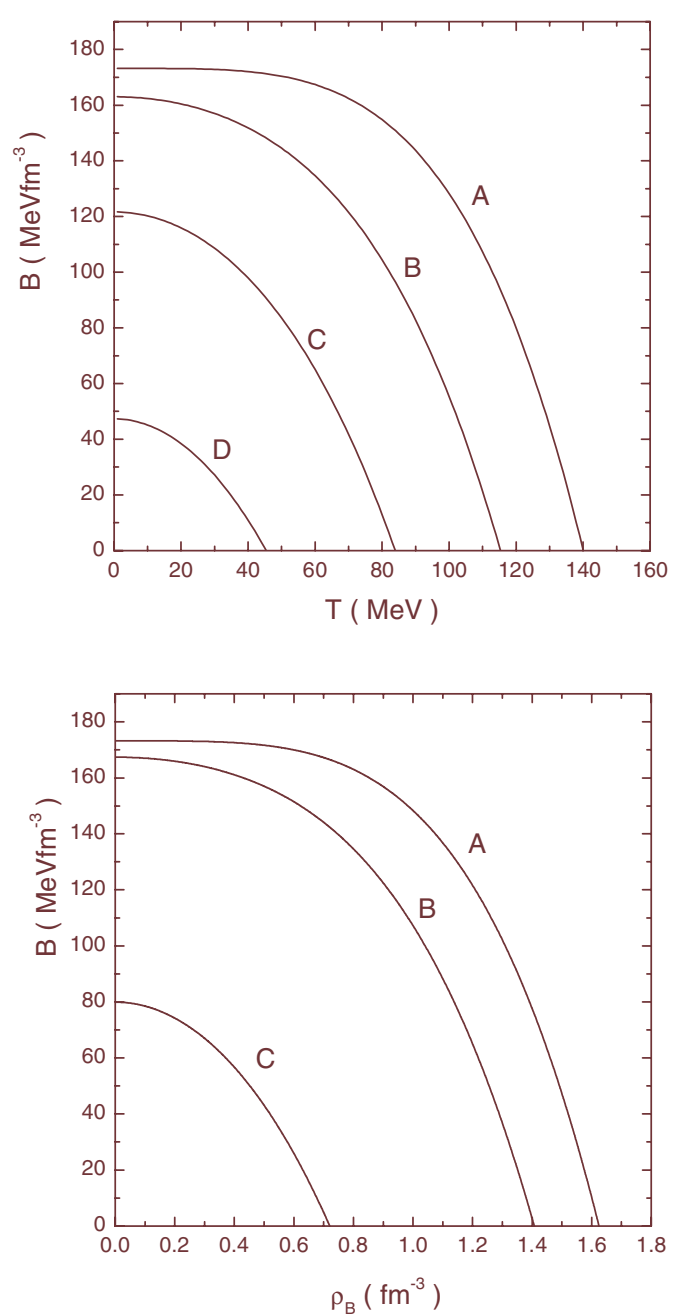

Fig. 1: Upper panel: bag constant vs. temperature at different nuclear matter density. A: $\rho_{B}=0.0 \mathrm{fm}^{-3}, \mathrm{~B}: \rho_{B}=0.8 \mathrm{fm}^{-3}$, C: $\rho_{B}=1.2 \mathrm{fm}^{-3}, \mathrm{D}: \rho_{B}=1.5 \mathrm{fm}^{-3}$. Lower panel: bag constant vs. nuclear matter density at different temperature. A: $T=$ $0 \mathrm{MeV}, \mathrm{B}: T=60 \mathrm{MeV}$, C: $T=120 \mathrm{MeV}$.

phenomenological Friedberg-Lee model, predicts a firstorder deconfinement phase transition for the full phase diagram. This is due to the fact that at the critical $T$ and $\rho_{B}$ the two vacuum states, namely the perturbative vacuum state and the physical vacuum state, appear with the same energy. This result differs from the predictions based on lattice gauge theory where a more complicated phase diagram on the $T-\rho_{B}$ plane has been obtained, giving more fruitful behaviors of the QCD phase $[19,20]$. For example, at finite temperature and zero chemical potential, the phase transition could be of second order; moreover, it is predicted that there may exist a critical point along the critical line.

At the end of paper, we have provided a comparison of the results obtained from the IQMDD model with the recent results obtained in the framework of effective four-fermion interaction theories. The recent efforts [20-29] have made very fruitful progress on the

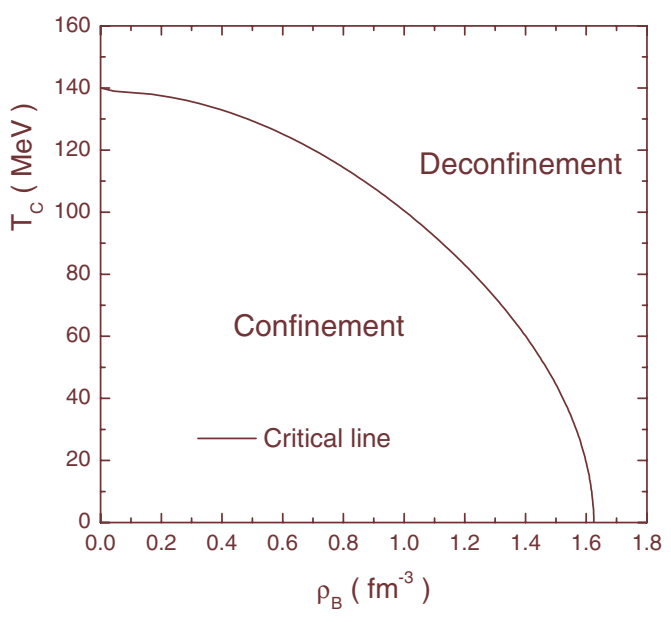

Fig. 2: Phase diagram of deconfinement in the IQMDD model.

QCD phase structure including finite-size hadron and magnetic effects. Their theoretical frame of the field theory is the imaginary-time temperature field theory, the same as for the IQMDD model. As the IQMDD model lacks chirality, we cannot predict a critical point along the critical line. However, we should emphasize that the IQMDD model can not only explain the quark deconfinement phase transition, but also successfully describe the saturation properties of the nuclear matter. In the IQMDD model, the symmetry nuclear matter is treated as uniform and unlimited, the vector-like interaction $g_{\omega}^{q} \psi^{+} \gamma^{\mu} \omega_{\mu} \psi$ between quarks and $\omega$-meson gives a contribution to an effective chemical potential of quarks, and the expected value of the $\omega$-meson is connected with the baryon density. Our numerical results show that the deconfining baryon density at zero temperature is about $1.63 \mathrm{fm}^{-3}$, which is very close to the value predicted by the lattice QCD theory. Meanwhile, the deconfining temperature at zero baryon density is $139 \mathrm{MeV}$ which is also reasonable compared to $170 \mathrm{MeV}$ given by the lattice QCD theory [33]. Recently, the Science journal [34] reported that the deconfining critical temperature for the QCD phase transition at zero baryon density is about $175 \mathrm{MeV}$.

In conclusion, we investigate the quark deconfinement phase transition at finite temperature and finite density of symmetric nuclear matter in the IQMDD model. We would like to emphasize that this is the basic advantage for the IQMDD model, because the saturation properties can be explained not only by the IQMDD model but also by the QMC model. The reason why quark deconfinement can be explained by the IQMDD model is that MIT boundary constraint has been dropped out and interactions between quark and mesons have been extended to the whole space. Instead of the MIT bag in the QMC model, a FL soliton bag is introduced in the IQMDD model, which makes it possible to discuss the quark deconfinement phase transition. The spontaneously breaking symmetry 
of the nonlinear $\sigma$-field is restored and the soliton bag will disappear at a critical condition.

The authors wish to thank Prof. RU-KEnG Su for useful correspondence. This work is supported by the National Natural Science Foundation of China (Grants 11105072 and 11035009).

\section{REFERENCES}

[1] Guichon P. A. M., Phys. Lett. B, 200 (1988) 235.

[2] Saito K., Tsushima K. and Thomas A. W., Prog. Part. Nucl. Phys., 58 (2007) 1 and references therein.

[3] Saito K. and Thomas A. W., Phys. Lett. B, 327 (1994) 9.

[4] Balian R. and Block C., Ann. Phys. (N.Y.), 60 (1970) 401.

[5] Wu C., Qian W. L. and Su R. K., Phys. Rev. C, 72 (2005) 035205.

[6] Wu C., Qian W. L. and Su R. K., Phys. Rev. C, 77 (2008) 015203.

[7] Fowler G. N., Raha S. and Weiner R. M., Z. Phys. C, 9 (1981) 271.

[8] Chakrabarty S., Raha S. and Sinha B., Phys. Lett. $B, 229$ (1989) 112.

[9] Benvenuto O. G. and Lugones G., Phys. Rev. D, 51 (1995) 1989; Lugones G. and Benvenuto O. G., Phys. Rev. D, 52 (1995) 1276.

[10] Zhang Y., Su R. K., Ying S. Q. and Wang P., Europhys. Lett., 53 (2001) 361.

[11] Zhang Y. and Su R. K., Phys. Rev. C, 65 (2002) 035202; 67 (2003) 015202.

[12] Zhang Y. and Su R. K., J. Phys. G, 30 (2004) 811.

[13] Friedberg R. and Lee T. D., Phys. Rev. D, 15 (1977) 1694; 16 (1977) 1096; 18 (1978) 2623.

[14] LeE T. D., Particle Physics and Introduction to Field Theory (Harwood Academic, New York) 1981.

[15] Wu C. and Ren Z., J. Phys. G, 37 (2010) 105110.
[16] Mao H., Su R. K. and Zhao W. Q., Phys. Rev. C, 74 (2006) 055204.

[17] Wu C. and Su R. K., J. Phys. G, 36 (2009) 095101.

[18] Gross D. J. and Neveu A., Phys. Rev. D, 10 (1974) 4410.

[19] Khanna F. C., Malbouisson A. P. C., Malbouisson J. M. C. and SAntanA A. E., Thermal Quantum Field Theory: Algebraic Aspects and Applications (World Scientific, Singapore) 2009.

[20] Abreu L. M., Malbouisson A. P. C. and Malbouisson J. M. C., EPL, 90 (2010) 11001.

[21] Khanna F. C., Malbouisson A. P. C., Malbouisson J. M. C. and Santana A. E., EPL, 92 (2010) 11001.

[22] Khanna F. C., Malbouisson A. P. C., Malbouisson J. M. C. and Santana A. E., EPL, 97 (2012) 11002.

[23] Abreu L. M., Malbouisson A. P. C., Malbouisson J. M. C. and Santana A. E., Nucl. Phys. B, 819 (2009) 127.

[24] Kneur J., Pinto M. B., Ramos R. O. and Staudt E., Phys. Rev. D, 76 (2007) 045020.

[25] Boenmer C., Fritsch U., Kraus S. and Thies M., Phys. Rev. D, 78 (2008) 065043.

[26] Abreu L. M., Malbouisson A. P.C. and Malbouisson J. M. C., Phys. Rev. D, 83 (2011) 025001.

[27] Abreu L. M., Malbouisson A. P. C. and Malbouisson J. M. C., Phys. Rev. D, 84 (2008) 065036.

[28] Malbouisson A. P. C., Malbouisson J. M. C., Santanab A. E. and Da Silva J. C., Phys. Lett. B, 583 (2004) 373.

[29] Khanna F. C., Malbouisson A. P. C., Malbouisson J. M. C., Queiroz H., Rocha-Filho T. M., Santanae A. E. and DA Silva J. C., Phys. Lett. B, 624 (1988) 316.

[30] Serot B. D. and Walecka J. D., Int. J. Mod. Phys. E, 6 (1997) 515 and references therein.

[31] Dolan L. and Jackiw R., Phys. Rev. D, 9 (1974) 3320.

[32] Kapusta J. I. and Gale C., Finite-Temperature Field Theory: Principles and Applications (Cambridge University Press, Cambridge) 2006.

[33] Rischke D. H., Prog. Part. Nucl. Phys., 52 (2004) 197.

[34] Gupta S., Luo X., Mohanty B., Ritter H. G. and Xu N., Science, 332 (2011) 1525. 